\title{
Local structure of amorphous and nanoscale systems by numerical XANES calculations
}

\author{
Yuanpeng Zhang ${ }^{1 \star}$, Osman Ersoy, Ali Karatutlu1, ${ }^{1}$, Andrei Sapelkin ${ }^{1}$ \\ ${ }^{1}$ School of Physics and Astronomy, Queen Mary, University of London, Mile End Road, \\ London E1 4NS, UK. \\ ${ }^{2}$ Electrical and Electronics Engineering, Yildirim Campus, Bursa Orhangazi University, \\ 16245 Yildirim, Bursa, Turkey. \\ Correspondence e-mail: a.sapelkin@qmul.ac.uk
}

\begin{abstract}
:
We examined sensitivity of the x-ray absorption near edge structure (XANES) to the local atomic arrangements in amorphous GaSb and nanoscale Ge. We demonstrate that XANES can be reliably used to obtain information about the local symmetry in these systems where lack of periodicity precludes the use of powerful diffraction methods. We used well-studied bulk amorphous GaSb as a reference to verify our approach and then extended it to matrixfree Ge nanoparticles. We show that in small $(\sim 4 \mathrm{~nm}) \mathrm{Ge}$ nanoparticles prepared by colloidal synthesis where there is evidence for structural disorder, the local tetrahedral (diamond-type) symmetry is still preserved out to the second coordination shell on average. We find the XANES data collected in transmission and optical detection modes for Ge nanoparticles show distinctive differences with the bulk samples and attribute this to particles size and surface effects. This approach is thus capable of delivering structural information on local symmetry, structure and morphology in nanoscale systems.
\end{abstract}

\section{Introduction}

Ever since the emergence of amorphous materials understanding their atomic structure and development of corresponding methods has received considerable fundamental and theoretical interest [1, 2]. A wide variety of $x$-ray and neutron scattering experimental methodologies has been developed that provided information required for structural analysis methods. Meanwhile, a number of theoretical techniques including the molecular dynamics quenching method [3-6], the WWW method [7,8], and several other improved activationrelaxation techniques [9-11], were developed to simulate amorphous systems of different types for further theoretical analysis of their structural. However, even today when the field of experimental techniques for characterizing the structures of amorphous materials is advanced, it still retained several fundamental challenges. The traditional powder X-ray or neutron diffraction (XRD and ND) provide limited structural information due to the lack of long-range correlations. Methods based on analysis of pair distribution function (PDF) made significant progress [12-14], but serious difficulties remain in the analysis of polyatomic systems. Total scattering technique [15] can in principle yield information about both shortrange and long-range order, but experiments are challenging and significant problems are encountered when the system is complex involving many distinctive chemical sites. Crucially, majority of these problems are translated into characterisation of a relatively new class of non-periodic systems - nanomaterials where the size and surface effects $[16,17]$ can affect sample morphology as well as phase stability and add further to the complexity of structural characterization.

There are several alternatives to XRD and ND techniques for characterization of amorphous and nano-sized materials. Transmission electron microscopy (TEM) and Raman are widely used for characterisation of nanoparticles. However, TEM and Raman suffer from the problem of sample annealing [16-21]. Furthermore, Raman data interpretation can be ambiguous due to difficulties in separating contributions from structural disorder and sizerelated affects. On the other hand, x-ray absorption spectroscopy (XAS) has long been recognised as a powerful alternative to diffraction and PDF based techniques because of its 
sensitivity to the local structure [22-24]. Most importantly, XAS offers a potential advantage over PDF methods, when considering the site selectivity of X-ray absorption. Indeed, since the development of the modern theory of the XAS, the extended part (EXAFS, between 50$1000 \mathrm{eV}$ above an x-ray absorption edge) of the overall signal has been successfully used in characterizing both bulk [25-29] and nano-systems [30-35]. This success is due to important and unique structural information EXAFS can yield including coordination numbers, interatomic distances, dynamic and static disorder around specific types of atoms in a multicomponent system. However, while analysis of EXAFS is a powerful method of choice for structural characterization, it provides virtually no information about the local symmetry of disordered samples due to limited (usually to the first coordination shell) structural content. This limitation is due to higher degree of structural disorder and because of the short (few $\AA$ ) electron mean free path of electrons with the energies in the EXAFS region. Furthermore, EXAFS analysis requires the model of the structure in question to be available in order to extract structural parameters. This makes it difficult (and in many cases impossible) to distinguish between metastable structures with similar (by $\sim 0.1-0.2 \AA$ ) local bond distances and coordination numbers when only first shell signal is available. In nanomaterials there are added problems of complex nanoscale morphology [36-38], possible contribution to the signal from metastable phases [16, 39, 40] and from surface effects.

One portion of the overall XAS signal - the X-ray absorption near-edge structure (XANES, extending up to around $50 \mathrm{eV}$ above an absorption edge) is frequently ignored as far as its structural content is concerned. At the same time, XANES is known to be very sensitive to the medium-range order and to the electronic properties of samples $[41,42]$. This is the consequence of the low kinetic energy of the photoelectron in the XANES region and hence relatively long electron mean free path $[43,44]$. This results in a significant contribution to XANES signal of multiple scattering effects that are sensitive to medium range order and can in principle deliver information about the local symmetry. Furthermore, the XANES part of the signal yields much higher signal-to-noise ratio compared to EXAFS and hence can be applicable, for example, to samples where satisfactory EXAFS data cannot be obtained.

Until very recently, for most part analysis of XANES has been qualitative and based on experimental standards owing to the complexity of information contained in XANES [45]. Recent developments in computational power and of the codes capable of sufficiently accurate XANES calculations make it possible to obtain quantitative structural and electronic information from experimental data. The currently available packages that allow the theoretical XANES calculation includes those based on multiple scattering theory in muffintin approximation (MTA) such as FEFF [46] and MXAN [47], those based on ground state density functional theory (DFT) such as WIEN2K [48]. DFT-based codes are computationally complex, while relatively fast and widely used MTA-based codes suffer from intrinsic problems associated with the approximation used. Since for the near-edge region, the energy of the photoelectron is comparable to the interstitial potential, the variation of potential in the interatomic space becomes crucial. Therefore, the less space is occupied by the muffin-tin radii, the more significant the errors associated with MTA calculations, which becomes even more of an issue for multiple scattering calculations. Thus, while MTA calculations may work sufficiently well for close-packed atomic systems (e.g. Cu, etc.) they become inadequate for covalently bonded open structures (e.g. Si, Ge, etc.). with the highly anisotropic charge density and relatively weak atomic scattering. In such a case recently developed FDMNES package [47] where in addition to MTA the finite difference method (FDM) is implemented resolves problems associated with MTA by using the local density approximation (LDA). Non-MTA XANES calculations has already been successfully employed on various glass and nano-systems [50-52] and FDMNES package had also been proved powerful form metallic [53] (in MTA mode) and metal oxide [54] (FDM mode) clusters.

In this report we focus on amorphous GaSb (aGaSb), crystalline GaSb (cGaSb) and Ge quantum dots (QDs) to test sensitivity of XANES to the local symmetry. The aGaSb is used as a reference system since it has been previously extensively studied $[55,56]$. 
Ge QDs, as group IV nanostructures that find potential application in multi-junction solar cells [57], photodetectors [58] and biological imaging [59]. These nanoscale systems show unusual optical [60] and structural [61] properties not found in bulk $\mathrm{Ge}$, while the relationship of the light emission to the underlying atomic (and electronic) structure is of the fundamental interest in the field of nanoscience and nanotechnology. Here we also utilised the optically detected XANES (OD-XANES) to look at the link between the local structure and the optical emission behaviour. OD-XAS signal is obtained by detecting the X-ray excited optical emission (XEOL) intensity and can thus provide a direct link between light emission and underlying structure. Furthermore, it has been observed [62-64] that in small nanoparticles the surface contribution to the Gibbs free energy may result in partial disorder and formation of metastable phases and there are virtually no experimental techniques that can give direct access to this level of structural information. Thus we also examine sensitivity of XANES to metastable phases.

\section{Methods}

For the analysis of GaSb XANES we used the XAS data obtained previously [55]. The oxygen-free Ge QDs (hydrogen-terminated) were obtained through stain etching, where the commercial Ge powder by Sigma-Aldrich (>99.999\%) was chemically etched in the solution of HF:H3PO4:H2O2 (15 mL:15 mL:0.075 mL, respectively). Following etching samples were transferred into silica capillaries in argon atmosphere and sealed to avoid exposure to air.

XAS measurements for Ge QDs were carried out in both transmission and optical detection modes at beamline B18 of Diamond Synchrotron Light Source. Crystalline Ge (cGe) reference was measured in transmission mode, while amorphous $\mathrm{Ge}(\mathrm{aGe})$ reference was taken from previously reported data [65].The data were collected at Ge K-edge using Si (111) monochromator with the energy resolution $\Delta E / E=1.4 \times 10^{-4}$. For the transmission mode detection, the ionization chambers were used to record the signal. The transmission mode measurement was carried out at room temperature. The OD-XAS experiments were conducted using X-ray excited optical luminescence (XEOL) signal. The detection system consisted of a Triax 190 spectrometer equipped with a Synapse CCD and a Newport VIS Femtowatt photoreceiver. A Hamamatsu R3809U-50 MCP photomultiplier was used for low signal conditions. The light was delivered to the spectrometer using an optical fibre. All the optical detection experiments have been conducted at $T=100 \mathrm{~K}$ using a cryojet system to improve light yield. The X-ray absorption signal was recorded by detecting the variation of integral intensity of the XEOL within the peak region (Fig. 1a) as a function of excitation energy. The mechanism of the OD- XAS is briefly illustrated in Fig. 1b. Electrons are first excited from the core level to the continuum and then through a complex relaxation process decay back to the valence band resulting in XEOL.

XAS data reduction and analysis were carried our using Demeter package [66]. The XANES spectra were calculated using FDMNES package [49]. The finite difference method (FDM) mode was used for XANES calculations. The cluster sizes used in XANES

calculations were set to $3.0 \AA$ (one coordination shell), $4.8 \AA$ (two shells) and $7 \AA$ (six shells), which includes 4, 29 and 71 atoms, respectively. The typical time scale for FDM calculations in parallel processing regime using five nodes for a $3 \AA$ zinc-blende GaSb cluster was around half an hour. This increased to 2-3 days for a $7 \AA$ cluster.

\section{Results and Discussion Crystalline and amorphous GaSb}

Bulk amorphous and crystalline were used as a reference to verify the capability of our approach to distinguish between two possible structural arrangements and to test sensitivity to the local symmetry. Samples of aGaSb are prepared by compression of cGaSb with the zinc-blende structure to above a structural transition, followed by fast decompression from the high pressure $\beta$-tin structure to ambient conditions [55]. Thus, potentially there could be two possible local configurations in the ambient amorphous phase: the metastable $\beta$-tin-like structure ( $\mathrm{Ga}$ and $\mathrm{Sb}$ occupy 6 -fold octahedral sites) and stable zinc-blende-like ( $\mathrm{Ga}$ and $\mathrm{Sb}$ 
occupy 4-fold tetrahedral sites) structure. It has been shown previously from by EXAFS analysis [55] that the bond angle in aGaSb is close to that of the zinc-blende structure, therefore the tetrahedral local symmetry is expected. To test that this can be reproduced in XANES calculations, we constructed the two corresponding structural models (zinc-blende [67] and $\beta$-tin [68]) calculated XANES signals for both structures and compared the results to the experimental data for $\mathrm{cGaSb}$ and aGaSb. The results for this calculations at Ga K-edge for cluster size of $7 \AA$ can be seen in Fig.2a. One can see that even with the largest cluster size only features $A, B, C, D$, but not feature $E$ (that can just be seen) are adequately reproduced suggesting that larger cluster size is required in case of cGaSb. The difference can be observed (see features A, B, D and E) between calculated XANES signals for zincblende and $\beta$-tin structures, with the zinc-blende simulated spectrum most closely corresponding to the experimental cGaSb data. This suggest that XANES calculations can reliably reproduce experimental data and distinguish between the phases.

Next, we had a closer look at XANES signal from aGaSb (see Fig. 2b). The shape of the signal is very different from that calculated for the $\beta$-tin-like structure (see Fig. 2a, b) and is rather similar to $c G a S b$, except the features are less pronounced. The latter is related to the structural disorder in aGaSb. At this stage we can conclude that tetrahedral local symmetry corresponding to the zinc-blende structure is preserved in aGaSb, which is in line with the previous [44] EXAFS analysis. Furthermore, armed with a capability to calculate XANES signals as a function of cluster size, we can also examine the range over which the local symmetry is preserved. In order to do that we compared calculated XANES spectra for several different cluster sizes: $3.0 \AA, 5.0 \AA$ and $7.0 \AA$. One can see that the main features $A$, $B, C$ and $D$ (for aGaSb, feature $E$ is missing in either experimental or theoretical spectra) observed both in $\mathrm{cGaSb}$ and aGaSb are only adequately reproduced for the largest cluster size of $7.0 \AA$ (see Fig. 2b) indicating that the short-range order (SRO) is extending to between $5.0 \AA$ and $7.0 \AA$. This indicates that numeric XANES analysis shows sufficient sensitivity to metastable phases and is capable of yielding information on the range over which the local order is preserved.

\section{Matrix-free Ge nanoparticles}

Having verified the methodology for numeric XANES calculations using a well-known system, we took advantage of the observed capabilities to study the local structure in small $(\sim 4 \mathrm{~nm})$ matrix-free Ge QDs. Following the route for the bulk GaSb system, the XANES spectra were calculated for Ge clusters with different phases reported previously: diamondtype tetrahedral (Dia) [62], body-centered BC8 [63] and simple tetragonal (ST12) [64]. The Ge-Ge first coordination shell distances in all these systems fall between $2.40 \AA$ and $2.49 \AA$ making it challenging to distinguish between the phases based on the first shell information derived from EXAFS. Following initial calculations for various cluster sizes, It was determined that the minimum cluster size of $7.0 \AA$ is required in order to observe key experimental spectral features. The XANES calculation results together with the experimental $\mathrm{cGe}$ reference data are shown in Fig. $3 a$. It can be seen that the main features $A, B$ and region $C$ (designated with the green rectangle) observed in the reference XANES of $\mathrm{cGe}$ can be reproduced in the calculated spectra corresponding to diamond-type $\mathrm{Ge}$ cluster. However, for BC8 structure the shape of the white line is clearly different, while for both $\mathrm{BC} 8$ and ST12 structures any features in the region $\mathrm{C}$ are absent. These results provide a positive test of XANES calculations against $\mathrm{CGe}$ reference and suggest sufficient sensitivity to distinguish between the metastable phases.

Following tests of calculations against a standard, we moved onto Ge QDs and analysed data collected in transmission and OD modes [69]. Transmission and OD-XANES spectra extracted from XEOL corresponding to the hydrogen-terminated Ge QDs are shown in Fig.3b. One can see that transmission and OD-XANES signals are very similar. When compared to diamond-type crystalline Ge reference (cGe, see Fig. 3b), the XANES spectra of the hydrogen-terminated Ge QDs are rather featureless suggesting a relatively high degree of disorder. However, features A, B and region $C$ can still be recognized. A 
comparison with the tetrahedral (diamond-type) amorphous $\mathrm{Ge}(\mathrm{aGe})$ reference [56] indicates similarity with the hydrogen-terminated sample. However, XANES of aGe reference sample is practically featureless except for the white line peak $A$, compared to the signal from $\mathrm{cGe}$ reference. For the hydrogen-terminated $\mathrm{Ge}$ nanoparticles feature $\mathrm{B}$ is visible in transmission, but less so in OD mode, while region $C$ feature can be observed (although quite weak) along with the main feature A. These observations indicate that hydrogenterminated Ge nanoparticles retain some degree of local order compared to the aGe reference. It is also clear from OD-XANES data that a pure Ge diamond-like structure is responsible for the light emission in the hydrogen-terminated sample.

To further explore the extent of local order in the hydrogen-terminated sample, we carried out numerical XANES calculations for diamond-type $\mathrm{Ge}(\mathrm{cGe})$ structure, utilizing the sensitivity of XANES to the local environment demonstrated in case of GaSb. Cluster sizes $3.0 \AA$ (only the first coordination shell), $4.8 \AA$ (two shells) and $7.0 \AA$ (six shells) were used for XANES calculations (see Fig. $3 \mathrm{~b}$ ) and the results are shown in Fig. 3b. One can see that cluster size of $3.0 \AA$ is sufficient to reproduce aGe signal, while at least $4.8 \AA$ cluster size is required to reproduce main features $(A, B$ and $C)$ observed in the hydrogen-terminated sample. Calculations for $7.0 \AA$ cluster reproduce not only all the main features, but also their position on the energy scale. These results suggest that the local diamond-type symmetry is retained at least out to the second coordination shell in the hydrogen-terminated Ge QDs. This is consistent with our previous findings (using a combination of OD-EAXFS and molecular dynamics simulations) showing that optical emission in hydrogen-terminated $\mathrm{Ge}$ QDs comes from the disordered surface layer ( $5 \AA$ in thickness) [60].

Thus we show that key spectral features can be adequately reproduced by XANES calculations using FDM mode in FDMNES. However, one can see that in case of Ge QDs the intensity of the white line (feature A in Fig. $3 b$ ) is not accurately reproduced for XANES signal collected transmission and OD modes. We believe that the origin of this discrepancy lies in the influence of particle size and surface effects on the electronic density of states above the Fermi level. In particular, it has been pointed out that hydrogen surface bonding has been found consistently to broaden and reduce the intensity of the white line in a number of catalysts [70]. This reduction has been attributed to a combination of the lattice contraction for small nanoparticles and of electron transfer from hydrogen and the effect on the electron density of states above the Fermi level. Furthermore, the feature $C$ in in ODXANES of Ge QDs appears to be less pronounced than that in the transmission data (see Fig. 3b) and accompanied by slightly more depressed white line. This suggest that ODXANES signal is associated with the structure having larger degree of disorder and relatively perhaps a higher proportion of hydrogen than that associated with the transmission data. This hypothesis is consistent with our previous findings [60] where disordered surface layer has been suggested to provide crucial contribution to the light emission. This sensitivity of OD-XANES to a distinctive subset of structures compared to the transmission measurements requires further investigation.

In this work calculations have been carried out for crystalline clusters to simulate $\mathrm{SRO}$ effects and this is the origin of the discrepancies described above. Using crystalline clusters places limitations on providing meaningful numerical comparisons (using chisquared test, for example) between simulated and experimental data. Ideally, XANES calculations using a realistic (disordered and hydrogen-terminated) cluster are needed. Such calculations require an adequate initial model with an ability to iterate XANES calculations as cluster structure is changed until the best fit to the experimental data is obtained. We believe that today such methodology can potentially be implemented, for example, within the framework of existing codes (e.g. RMCprofile [71]).

To summarise, we found that XANES shows sufficient sensitivity to the local symmetry in systems with large disorder to distinguish between a number of possible metastable phases and thus to determine the local symmetry. We also show that XANES signal strongly depends on the cluster size used in calculations, thus enabling to provide a numeric estimate for the extend of the SRO in amorphous and low-dimensional systems. 


\section{Conclusion}

We demonstrated a XANES-based methodology to obtain information about the local symmetry in disordered systems and to discriminate between possible metastable phases. This methodology was tested on a well-studied system used as a reference - bulk amorphous GaSb. We further applied this methodology to a novel system - small matrix-free Ge nanoparticles. We used the data collected in an optically-detected mode (which allows to link directly the light emission with the contributing structure) and in a transmission mode. We also show that this approach can provide numerical information about the spatial range beyond which the local structural order is lost. We found that in hydrogen-terminated Ge nanoparticles OD-XANES signal can be satisfactory modelled based on the diamond-type structure with the local ordering extending to between $4.8 \AA$ and $7.0 \AA$. Our findings suggest that numerical XANES calculations can be used as a tool to probe the local symmetry in nanoscale structures and disordered and metastable systems in general.

\section{Acknowledgement}

XANES calculations were carried out using Queen Mary's MidPlus computational facilities, supported by QMUL Research-IT and funded by EPSRC grant EP/K000128/1. We would like to thank Diamond Light Source for the beamline (B18) and the experimental support from Konstantin Ignatyev, Giannantonio Cibin and Diego Gianolio etc. AK and OE acknowledge the Turkish Ministry of National Education. $Y Z$ is grateful to the Chinese Scholarship Council (CSC) for financial support of his PhD study.

\section{References}

[1] G.T. Barkema, N. Mousseau, Event-based relaxation of continuous disordered systems, Phys. Rev.Lett., 77 (1996) 4358-4361.

[2] W.H. Zachariasen, The atomic arrangement in glass, J. Am. Chem. Soc., 54 (1932) 3841-3851.

[3] E. Fois, A. Selloni, G. Pastore, Q.M. Zhang, R. Car, STRUCTURE, ELECTRONICPROPERTIES, AND DEFECTS OF AMORPHOUS GALLIUM-ARSENIDE, Phy. Rev. B, 45 (1992) 13378-13382.

[4] M. Ishimaru, S. Munetoh, T. Motooka, Generation of amorphous silicon structures by rapid quenching: A molecular-dynamics study, Phys. Rev. B, 56 (1997) 15133-15138.

[5] C. Molteni, L. Colombo, L. Miglio, STRUCTURE AND PROPERTIES OF AMORPHOUS GALLIUM-ARSENIDE BY TIGHT-BINDING MOLECULAR-DYNAMICS, Phys. Rev. B, 50 (1994) 4371-4377.

[6] H. Seong, L.J. Lewis, Tight-binding molecular-dynamics study of density-optimized amorphous GaAs, Phys. Rev. B, 53 (1996) 4408-4414.

[7] N. Mousseau, G.T. Barkema, Fast bond-transposition algorithms for generating covalent amorphous structures, Curr. Opin. Solid State \& Mater. Sci., 5 (2001) 497-502.

[8] F. Wooten, K. Winer, D. Weaire, COMPUTER-GENERATION OF STRUCTURAL MODELS OF AMORPHOUS SI AND GE, Phys. Rev. Lett., 54 (1985) 1392-1395.

[9] G.T. Barkema, N. Mousseau, Event-based relaxation of continuous disordered systems, Phys. Rev. Lett., 77 (1996) 4358-4361.

[10] G.A. Connell, R.J. Temkin, Modeling the structure of amorphous tetrahedrally coordinated semiconductors, Phys. Rev. B., 9 (1974) 5323-5326.

[11] N. Mousseau, L.J. Lewis, Topology of amorphous tetrahedral semiconductors on intermediate length scales, Phys. Rev. Lett., 78 (1997) 1484-1487.

[12] S.D.M. Jacques, M. Di Michiel, S.A.J. Kimber, X. Yang, R.J. Cernik, A.M. Beale, S.J.L. Billinge, Pair distribution function computed tomography, Nat. Comm., 4 (2013) 2536.

[13] C.D. Martin, S.M. Antao, P.J. Chupas, P.L. Lee, S.D. Shastri, J.B. Parise, Quantitative high-pressure pair distribution function analysis of nanocrystalline gold, Appl. Phys. Lett., 86 (2005) 061910. 
[14] C.A. Young, A.L. Goodwin, Applications of pair distribution function methods to contemporary problems in materials chemistry, J. Mater. Chem., 21 (2011) 6464-6476.

[15] M. T. Dove, M. G. Tucker, D. A. Keen, Neutron total scattering method: simultaneous determination of long-range and short-range order in disordered materials, Eur. J. Mineral., 14, (2002), 331-348.

[16] H.W. Chiu, S.M. Kauzlarich, E. Sutter, Thermal behavior and film formation from an organogermanium polymer/nanoparticle precursor, Langmuir, 22 (2006) 5455-5458.

[17] D. Tomanek, M.A. Schluter, STRUCTURE AND BONDING OF SMALL

SEMICONDUCTOR CLUSTERS, Phys. Rev. B, 36 (1987) 1208-1217.

[18] N.H. Chou, K.D. Oyler, N.E. Motl, R.E. Schaak, Colloidal Synthesis of Germanium Nanocrystals Using Room-Temperature Benchtop Chemistry, Chem. Mater., 21 (2009) 4105-4107.

[19] C.A. Moore, J.J. Rocca, T. Johnson, G.J. Collins, P.E. Russell, LARGE AREA ELECTRON-BEAM ANNEALING, Appl. Phys. Lett., 43 (1983) 290-292.

[20] S.-G. Ryu, I. Gruber, C.P. Grigoropoulos, D. Poulikakos, S.-J. Moon, Large area crystallization of amorphous Si with overlapping high repetition rate laser pulses, Thin Solid Films, 520 (2012) 6724-6729.

[21] W. Tseng, H. Dietrich, J. Davey, A. Christou, W.T. Anderson, THE EFFECTS OF IONIMPLANTATION AND PULSED ELECTRON-BEAM ANNEAL ON GE FILMS GROWN EPITAXIALLY ON (100) GAAS, J. Electr. Mater., 9 (1980) 685-692.

[22] T.J. Penfold, C.J. Milne, M. Chergui, RECENT ADVANCES IN ULTRAFAST X-RAY ABSORPTION SPECTROSCOPY OF SOLUTIONS, in: S.A. Rice, A.R. Dinner (Eds.) Advances In Chemical Physics, Vol 153, 2013, pp. 1-41.

[23] H. Wende, Recent advances in x-ray absorption spectroscopy, Rep. Progr. Phys., 67 (2004) 2105-2181.

[24] J. Yano, V.K. Yachandra, X-ray absorption spectroscopy, Photosynth. Res., 102 (2009) 241-254.

[25] F. Jiao, A. Harrison, J.C. Jumas, A.V. Chadwick, W. Kockelmann, P.G. Bruce, Ordered mesoporous Fe2O3 with crystalline walls, J. Am. Chem. Soc., 128 (2006) 5468-5474.

[26] S.T. Oyama, Novel catalysts for advanced hydroprocessing: transition metal phosphides, J. Catal., 216 (2003) 343-352.

[27] I.E. Wachs, Recent conceptual advances in the catalysis science of mixed metal oxide catalytic materials, Catal. Today, 100 (2005) 79-94.

[28] G. N. Greaves, EXAFS and the structure of glass, J. Non-Cryst. Solids, 71 (1985) 203217.

[29] G. N. Greaves, A. Fontaine and P. Lagarde and D. Raous and S. J. Gurman, Nature, 293 (1981) 611-616.

[30] X.L. Li, G. Pan, Y.W. Qin, T.D. Hu, Z.Y. Wu, Y.N. Xie, EXAFS studies on adsorptiondesorption reversibility at manganese oxide-water interfaces II. Reversible adsorption of zinc on delta-MnO2, J. Coll. Interf.Sci., 271 (2004) 35-40.

[31] S.C. Liou, S.Y. Chen, H.Y. Lee, J.S. Bow, Structural characterization of nano-sized calcium deficient apatite powders, Biomaterials, 25 (2004) 189-196.

[32] F. Maillot, G. Morin, Y.H. Wang, D. Bonnin, P. Ildefonse, C. Chaneac, G. Calas, New insight into the structure of nanocrystalline ferrihydrite: EXAFS evidence for tetrahedrally coordinated iron(III), Geochim. Cosmochim. Acta, 75 (2011) 2708-2720.

[33] G. Pan, Y.W. Qin, X.L. Li, T.D. Hu, Z.Y. Wu, Y.N. Xie, EXAFS studies on adsorptiondesorption reversibility at manganese oxides-water interfaces I. Irreversible adsorption of zinc onto manganite (gamma-MnOOH), J. Coll. Interf. Sci., 271 (2004) 28-34.

[34] M. Sasaki, M. Osada, N. Sugimoto, S. Inagaki, Y. Fukushima, A. Fukuoka, M. Ichikawa, Novel templating fabrication of nano-structured Pt clusters and wires in the ordered cylindrical mesopores of FSM-16 and their unique properties in catalysis and magnetism, Microp. Mesop. Mater., 21 (1998) 597-606.

[35] Y. Zhang, O. Ersoy, A. Karatutlu, W. Little, A. Sapelkin, Local structure of Ge quantum dots determined by combined numerical analysis of EXAFS and XANES data, J.

Synchrotron Radiat., 23 (2016) 253-259. 
[36] R. Ke, X. Zhang, L. Wang, C. Zhang, S. Zhang, H. Niu, C. Mao, J. Song, B. Jin, Y. Tian, Enhanced electrochemiluminescence of CdSe quantum dots coupled with MoS2-chitosan nanosheets, J. Solid State Electrochem., 19 (2015) 1633-1641.

[37] A. Samavati, M.K. Mustafa, Z. Othaman, S.K. Ghoshal, Ge Nanoislands Grown by Radio Frequency Magnetron Sputtering: Comprehensive Investigation of Surface Morphology and Optical Properties, J. Nanomater., (2015) 681242.

[38] A. Samavati, Z. Othaman, S.K. Ghoshal, M.K. Mustafa, Effects of annealing temperature on shape transformation and optical properties of germanium quantum dots, Chin. Phys. B, 24 (2015) 028103.

[39] R.J. Nelmes, M.I. McMahon, N.G. Wright, D.R. Allan, J.S. Loveday, STABILITY AND CRYSTAL-STRUCTURE OF BC8 GERMANIUM, Phys. Rev. B, 48 (1993) 9883-9886. [40] D. Selli, I.A. Baburin, R. Martonak, S. Leoni, Novel metastable metallic and semiconducting germaniums, Sci. Rep., 3 (2013) 1466.

[41] D. Norman, X-RAY ABSORPTION-SPECTROSCOPY (EXAFS AND XANES) AT SURFACES, J. Phys. C-Solid State Phys., 19 (1986) 3273-3311.

[42] S. Pin, V. Letilly, B. Alpert, R. Cortes, XANES SPECTROSCOPY SENSITIVITY TO SMALL ELECTRONIC CHANGES - CASE OF CARP AZIDOMETHEMOGLOBIN, FebS Lett., 242 (1989) 401-404.

[43] M. Newville, Fundamentals of XAFS, in: G.S. Henderson, D.R. Neuville, R.T. Downs (Eds.) Spectroscopic Methods In Mineralology And Materials Sciences, 2014, pp. 33-74. [44] J.E. Penner-Hahn, Applications of X-ray absorption spectroscopy to inorganic and bioinorganic chemistry, Abstr. Pap. Amer. Chem. Soc., 224 (2002) U149-U149.

[45] G. Bunker, Introduction to XAFS: A Practical Guide to X-ray Absorption Fine Structure, Cambridge University Press, UK, 2010.

[46] J. J. Rehr, J. J. Kas, F. D. Vila, M. P. Prange, K. Jorissen, Parameter-free calculations of X-ray spectra with FEFF9, Phys. Chem. Chem. Phys., 12 (2010) 5503-5513.

[47] M. Benfatto, A. Congiu-Castellano, A. Daniele, S. D. Longa, MXAN: a new software procedure to perform geometrical fitting of experimental XANES spectra, J. Synchrotron Radiat. 8 (2001) 267-269.

[48] P. Blaha, K. Schwarz, G. Madsen, D. Kvasnicka, J. Luitz, WIEN2K, an augmented plane wave+local orbitals program for calculating crystal properties, Karlheinz Schwarz, Techn. Universität Wien, Austria, 2001.

[49] Y. Joly, X-ray absorption near-edge structure calculations beyond the muffin-tin approximation, Phys. Rev. B, 63 (2001) 125120.

[50] N. Trcera, D. Cabaret, S. Rossano, Experimental and theoretical study of the structural environment of magnesium in minerals and silicate glasses using X-ray absorption nearedge structure, Phys. Chem. Mineral, 36 (2009) 241-257.

[51] N. Trcera, S. Rossano, K. Madjer, Contribution of molecular dynamics simulations and ab initio calculations to the interpretation of $\mathrm{Mg} \mathrm{K}$-edge experimental XANES in K2O-MgO3SiO(2) glass, 23 (2011) 255401.

[52] D. Cabaret, F. Mauri, G. S. Henderson, Oxygen K-edge XANES of germanates investigated using first-principles calculations, 75 (2007) 184205.

[53] C. Maurizio, T. Cesca, G. Perotto, B. Kalinic, N. Michieli, C. Scian, Y. Joly, G. Battaglin, P. Mazzoldi, G. Mattei, Core-shell-like Au sub-nanometer clusters in Er-implanted silica, Nanoscale, 7 (2015) 8968-8977.

[54] A. Tougerti, E. Berrier, A. S. Mamede, C. La Fontaine, V. Briois, Y. Joly, E. Payen, J. F. Paul, S. Cristol, Synergy between XANES spectroscopy and DFT to elucidate the amorphous structure of heterogeneous catalysts: TiO2-supported molybdenum oxide catalysts, Angew. Chem. Int. Ed. 52 (2013) 6440-6444.

[55] A.V. Sapelkin, S.C. Bayliss, A.G. Lyapin, V.V. Brazhkin, A.J. Dent, Structure of bulk amorphous GaSb: A temperature-dependent EXAFS study, Phys. Rev. B, 56 (1997) 1153111535.

[56] S.S. Claudia, C.R. Mark, X-ray absorption spectroscopy of semiconductors, Springer, USA, 2015. 
[57] W. Guter, J. Schone, S. P. Philipps, M. Steiner, G. Siefer, A. Wekkeli, E. Welser, E. Oliva, A. W. Bett, F. Dimroth, Appl. Phys. Lett., 94 (2009) 223504.

[58] S. Assefa, F. N. Xia, Y. A. Vlasov, Reinventing germanium avalanche photodetector for nanophotonic on-chip optical interconnects, Nature, 464 (2010) 80-U91.

[59] T. N. Lambert, N. L. Andrews, H. Gerung, T. J. Boyle, J. M. Oliver, B. S. Wilson, S. M. Han, Water-soluble germanium (0) nanocrystals: cell recongnition and near-infrared photothermal conversion properties, 3 (2007) 691-699.

[60] W. Little, A. Karatutlu, D. Bolmatov, K. Trachenko, A.V. Sapelkin, G. Cibin, R. Taylor, F. Mosselmans, A.J. Dent, G. Mountjoy, Structural origin of light emission in germanium quantum dots, Sci. Rep., 4 (2014) 7372.

[61] N. R. C. Corsini, Y. Zhang, W.R. Little, A. Karatutlu, O. Ersoy, P. D. Haynes, C. Molteni, N. D. M. Hine, I. Hernandez, J. Gonzalez, F. Rodriguez, V. V. Brazhkin, A. V. Sapelkin,

Pressure-Induced Amorphization and a New High Density Amorphous Metallic Phase in Matrix-Free Ge Nanoparticles, Nano Lett., 15 (2015), 7334-7340.

[62] A. Smakula, J. Kalnajs, Precision determination of lattice constants with a GeigerCounter X-ray diffractometer, Phys. Rev., 99 (1955) 1737-1743.

[63] R. J. Nelmes, M. I. McMahon, N. G. Wright, D. R. Allan, J. S. Loveday, Stability and crystal structure of BC8 germanium, Phys. Rev. B, 48 (1993) 9883-9886.

[64] A. Mujica, R. J. Needs, First-principles calculations of the structural propertie, stability, and band structure of complex tetrahedral phases of germanium: ST12 and BC8, Phys. Rev. B, 48 (1993) 17010-17017.

[65] J.P. Itie, A. Polian, G. Calas, J. Petiau, A. Fontaine, H. Tolentino, Pressure-induced coordination changes in crystalline and vitreous GeO2, Phys. Rev. Lett., 63 (1989) 398-401. [66] M. Newville, B. Ravel, D. Haskel, J.J. Rehr, E.A. Stern, Y. Yacoby, ANALYSIS OF MULTIPLE-SCATTERING XAFS DATA USING THEORETICAL STANDARDS, Phys. B, 208 (1995) 154-156.

[67] M. E. Straumanis, C. D. Kin, Lattice parameters, thermal expansion coefficients, phase width, and perfection of the structure of GaSb and InSb, J. Appl. Phys., 36 (1965) 38223825.

[68] S. T. Weir, Y. K. Vohra and A. L. Ruoff, Phase transitions in GaSb to $110 \mathrm{GPa}(11$ Mbar), Phys. Rev. B. 36 (1987) 4543-4546.

[69] M. G. Dowsett, A. Adriaens, G. K. Jones, N. Poolton, S. Fiddy, S. Nikitenko, Optically detected X-ray absorption spectroscopy measurements as a means of monitoring corrosion layers on copper, Anal. Chem., 80 (2008) 8717-8724.

[70] Y. Lei, J. Jelic, L. C. Nitsche, R. Meyer, andJ. Miller Effect of Particle Size and Adsorbates on the $L_{3}, L_{2}$ and $L_{1}$ X-ray Absorption Near Edge Structure of Supported Pt Nanoparticles. Top Catal 54 (2011) 334-348

[71] M. G. Tucker, D. A. Keen, M. T. Dove, A. L. Goodwin and Q. Hui, RMCProfile: Reverse Monte Carlo for polycrystalline materials. Journal of Physics: Condensed Matter 19 (2007) art no 335218 (16 pp).

Figure. 1. (a) An example XEOL spectrum for hydrogen-terminated Ge QDs used to collect OD-XANES. (b) A schematic illustration of XEOL following X-ray absorption. (c) Atomic structure of diamond-type (Dia) Ge clusters used for XANES calculations.

Figure. 2. (a) Ga K-edge XANES for GaSb compound at $T=78 \mathrm{~K}$, together with the corresponding simulated XANES for zinc blende (ZB) and $\beta$-tin (BT) structures. (b) Ga Kedge XANES of both crystalline and amorphous $\mathrm{GaSb}$ compounds at $\mathrm{T}=78 \mathrm{~K}$, together with the corresponding simulated XANES spectra where the calculation cluster was set to $3.0 \AA$, $5.0 \AA$ and $7.0 \AA$, respectively. The key spectral features discussed in the text are marked $A$, $B, C, D$ and $E$. 
Figure. 3. (a) Comparison of XANES spectra for $c G e$ reference and the calculated spectra for Dia-Ge, BC8-Ge and ST12-Ge with cluster size of $7.0 \AA$. (b) The comparison of transmission and optically detected XANES spectra for hydrogen-terminated Ge QDs, together with crystalline and amorphous $\mathrm{Ge}$ [50] references. Calculated XANES data for diamond-type cluster sizes of $3.0 \AA, 4.8 \AA$ and $7.0 \AA$ are also shown. The key spectral features discussed in the text are marked $A, B$ and $C$ (marked with the green rectangle). 
Click here to download high resolution image

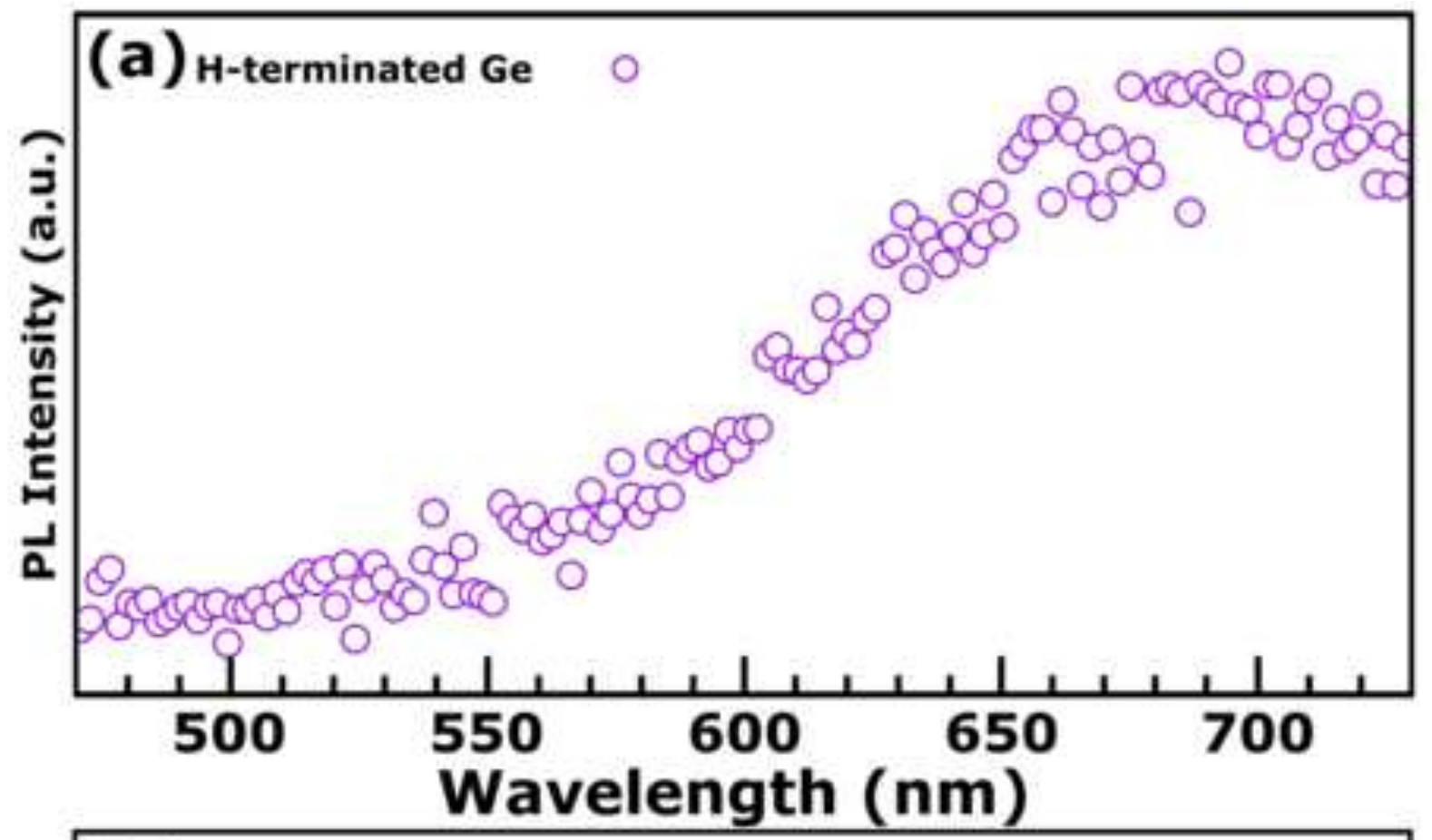

(c)

Dia-Ge Cluster

$3.0 \AA$

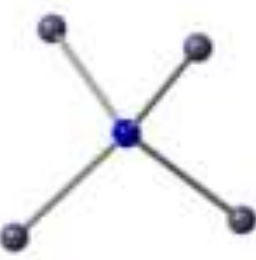

$4.8 \AA$
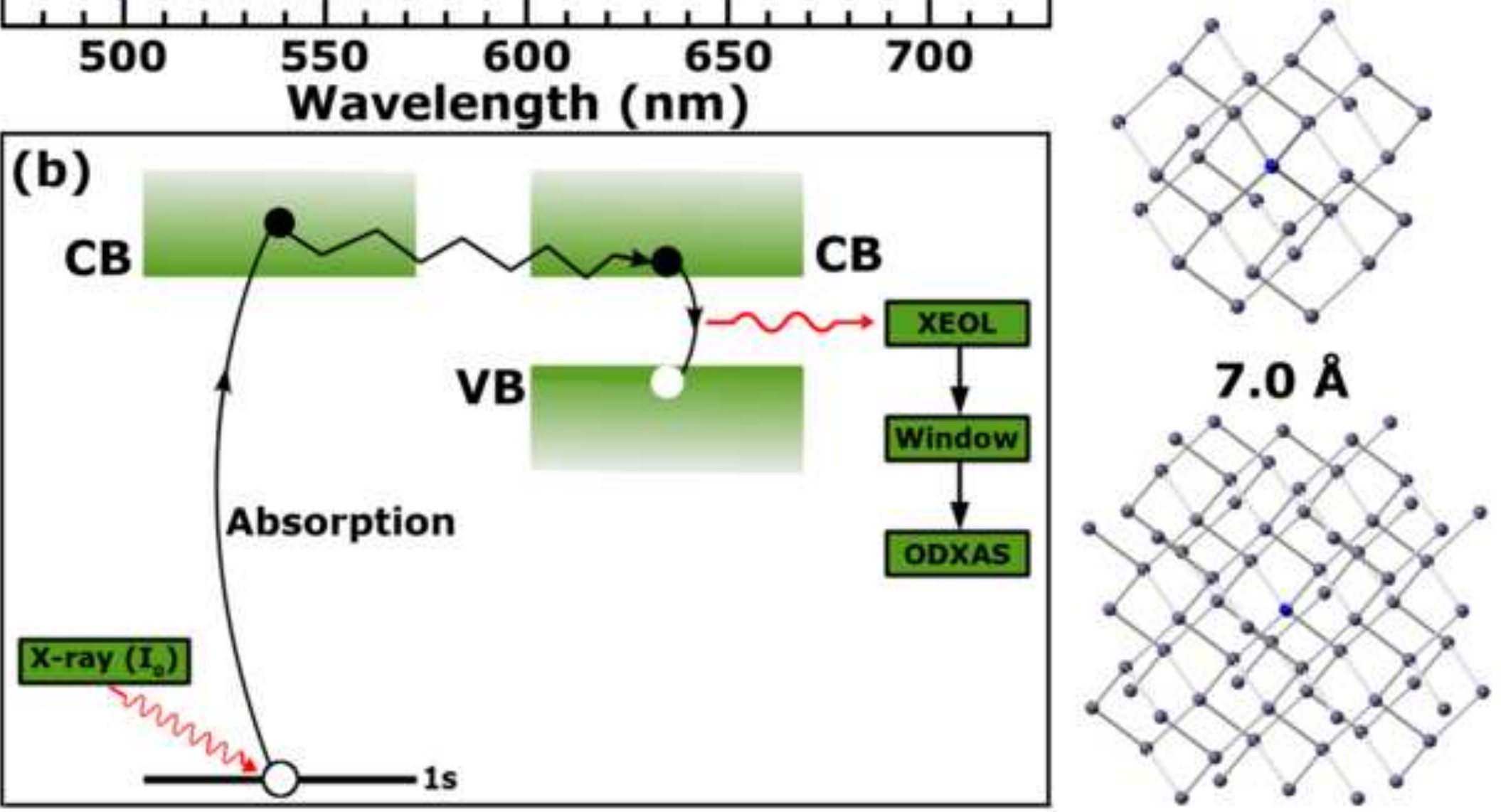


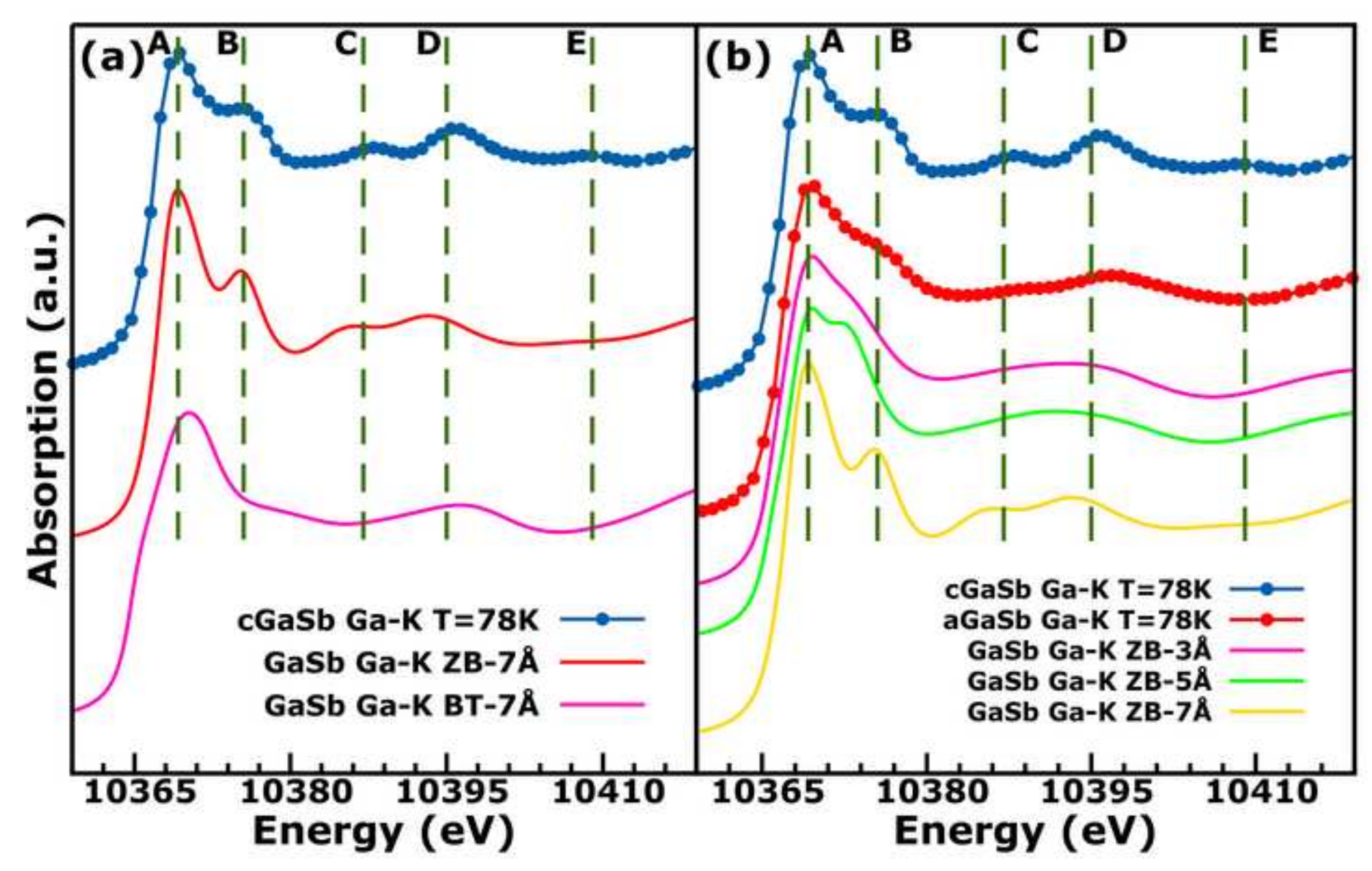




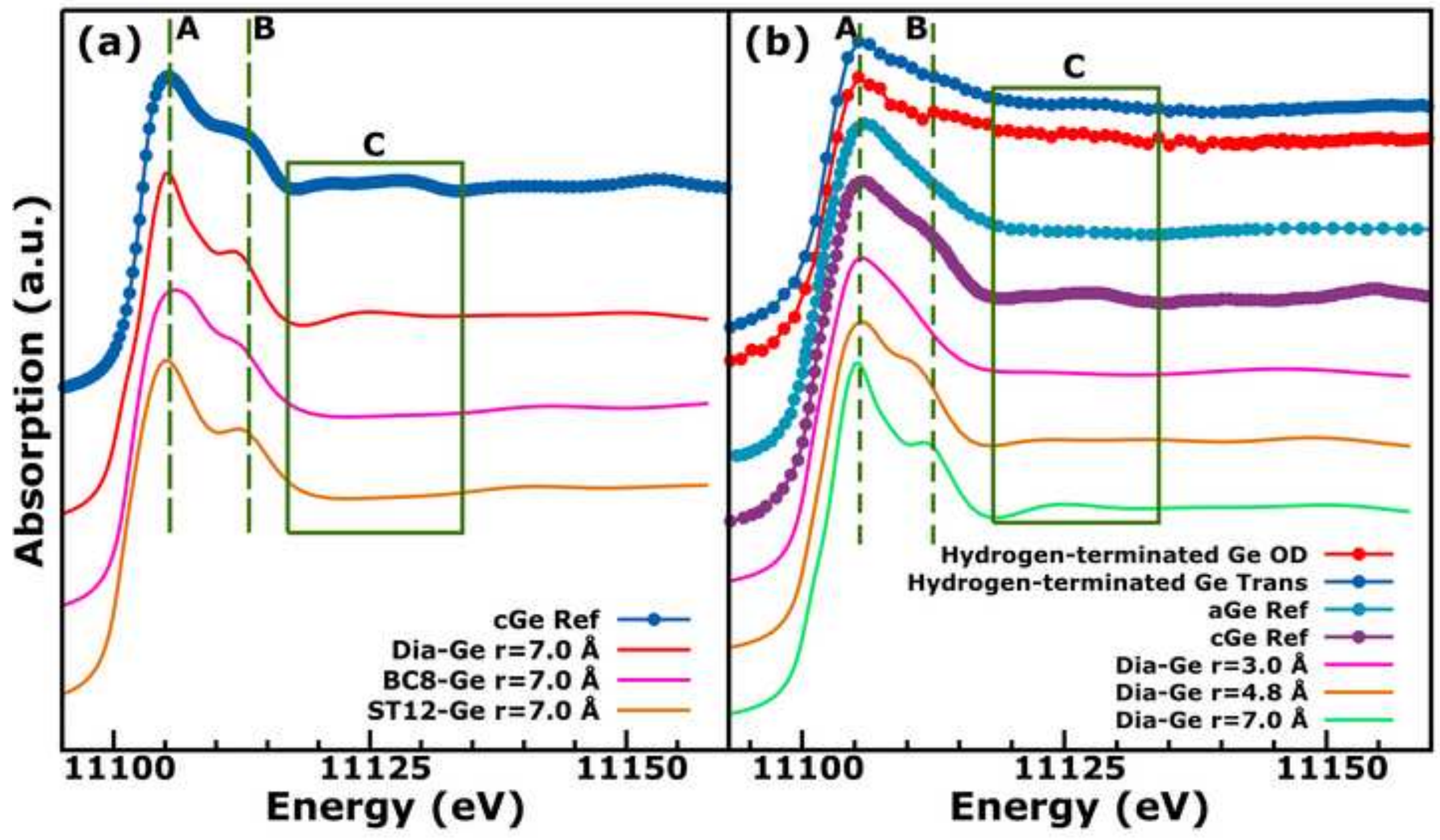

